\title{
Communication-Efficient MPC for General Adversary Structures
}

\author{
Joshua Lampkins ${ }^{1}$ and Rafail Ostrovsky ${ }^{2}$ \\ jlampkins@math.ucla.edu,rafail@cs.ucla.edu \\ ${ }^{1}$ Department of Mathematics, University of California, Los Angeles \\ 2 Department of Computer Science, University of California, Los Angeles
}

April 20, 2014

\begin{abstract}
A multiparty computation (MPC) protocol allows a set of players to compute a function of their inputs while keeping the inputs private and at the same time securing the correctness of the output. Most MPC protocols assume that the adversary can corrupt up to a fixed fraction of the number of players. Hirt and Maurer initiated the study of MPC under more general corruption patterns, in which the adversary is allowed to corrupt any set of players in some pre-defined collection of sets [6]. In this paper we consider this important direction of research and present significantly improved communication complexity of MPC protocols for general adversary structures. More specifically, ours is the first unconditionally secure protocol that achieves linear communication in the size of multiplicative Monotone Span Program representing the adversary structure in the malicious setting against any $Q 2$ adversary structure, whereas all previous protocols were at least cubic.
\end{abstract}

Keywords: Multiparty Computation, Secret Sharing, General Adversaries, Q2 Adversary Structures 


\section{Introduction}

In a multiparty computation (MPC) protocol, it is assumed that some of the players might collude together to attempt to determine some other player's input or to alter the output of the function. This is generally modeled as a single adversary corrupting a subset of the players. In order for a protocol to work, one must assume that the adversary is limited in the number of players he can corrupt. Most MPC protocols have a simple threshold requirement on the adversary. For instance, if the total number of players is $n$ and the number of players corrupted by the adversary is $t$, then a protocol might require $t<n / 3$ or $t<n / 2$.

In this paper, we consider requirements on the adversary which are more general than just threshold requirements. If $\mathcal{P}$ is the set of players, then the most general way of expressing the limitations of the adversary is to select a subset $\mathcal{A} \subset 2^{\mathcal{P}}$, called an adversary structure. The adversary is then allowed to corrupt any set of players in $\mathcal{A}$. This paper constructs a Multiparty Computation protocol that is secure against a malicious, adaptive adversary whose corruption pattern is specified by a general $Q 2$ adversary structure. $^{1}$

\subsection{Previous Work}

The first MPC protocol for general adversaries was given in Hirt and Maurer [6]. The protocol was recursive, relying on the use of virtual processors/players and "nesting" the virtualization. The MPC protocol for malicious $Q 2$ adversaries with a broadcast channel had communication complexity superpolynomial in the size of the description of the adversary structure; the protocol was slightly modified in Fitzi, Hirt and Maurer [5] to yield polynomial communication complexity. More explicit protocols were given in Cramer, Damgård and Maurer [3], Smith and Stiglic [11], and Cramer, Damgård, Dziembowski, Hirt and Rabin [4], each paper constructing an MPC protocol based on Monotone Span Program (MSP) secret sharing, initially developed by Karchmer and Wigderson [9]. An MPC protocol based on a different secret sharing scheme is given in Beaver and Wool [1], which deals with passive adversaries only.

This paper, along with [11] and [4], state the communication complexity in terms of the size $d$ of the smallest multiplicative $\mathrm{MSP}^{2}$ representing the adversary structure. A separate line of work (including [6] and [5] cited above) on MPC for general adversaries has stated the complexity in terms of the size of the set of maximal sets in the adversary structure, $|\overline{\mathcal{A}}|$. These works do not rely on MSPs, but construct fundamentally different secret sharing schemes. In [10] and [7], the authors achieve a protocol that is cubic in $|\overline{\mathcal{A}}|$; in $[8]$, the authors present a protocol linear in $|\overline{\mathcal{A}}|$.

The two lines of work, those stating the complexity in terms of $d$ and those stating the complexity in terms of $|\overline{\mathcal{A}}|$, are incomparable. To see why, consider that in [6], Hirt and Maurer describe a way of representing an arbitrary adversary structure $\mathcal{A}$ with a circuit of threshold gates, and in [3], it is shown how to construct a multiplicative MSP from such a circuit. Following that technique, one would construct an MSP of size $d=|\overline{\mathcal{A}}|^{\log _{3 / 2}(3)}=|\overline{\mathcal{A}}|^{2.7}$. So in that case, $d$ is higher than $|\overline{\mathcal{A}}|$.

However, the above only provides an upper bound on $d$. There are certain adversary structures for which the above procedure does not lead to the smallest MSP. In fact, $d$ can be as low as $n$. As a concrete example, suppose there is an MPC protocol being run by the U.S., China, and Russia. Each country has five servers running the protocol. We say that a country is corrupt if at least 3 out of the 5 servers in that country are corrupt. The MPC protocol tolerates an adversary that corrupts at most one country. In this case, $d=n=15$, whereas $|\overline{\mathcal{A}}|=3\left(\begin{array}{l}5 \\ 2\end{array}\right)^{2}=300$.

\subsection{Our Contributions}

This paper provides an MPC protocol in the setting of an malicious $Q 2$ adversary with a broadcast channel. We improve upon the amortized efficiency of previous protocols for malicious, $Q 2$ adversaries, as shown in

\footnotetext{
${ }^{1} Q 2$ adversary structures are defined in section 2 .

${ }^{2}$ The terms MSP and multiplicative MSP are defined in section 2.
} 


\begin{tabular}{||l|c|c|c|}
\hline Paper & {$[11]$} & {$[4]$} & This Paper \\
\hline Bandwidth & $\Omega\left(\mathcal{C} \kappa n d^{3}\right)$ & $\Omega\left(\mathcal{C} n d^{3}\right)$ & $O\left(\mathcal{C} n^{2} d+n^{3} d+\kappa n^{4} \log d\right)$ \\
\hline
\end{tabular}

Table 1: Comparison of MPC protocols secure against malicious, $Q 2$ adversaries with a broadcast channel. Here, $d$ is the size of the smallest multiplicative MSP representing adversary structure, $n$ is the number of players, $\mathcal{C}$ is the size of the circuit, and $\kappa$ is the security parameter. Bandwidth is measured in field elements, and counts both point-to-point communications and broadcasts.

Table 1. In examining the table note that $d$ is the dominating term and can be exponential in $n$ and is always at least $n$. So in addition to providing a strict improvement over previous protocols, our result is the first MPC protocol secure against malicious and adaptive $Q 2$ adversaries that has communication complexity linear in $d$.

\section{$1.3 \quad$ Techniques}

One way of dealing with disputes is with a technique called Kudzu shares, as first defined by BeerliováTrubíniová and Hirt [2]. When one player accuses another of lying (or other such misbehavior), they are said to be in dispute. When a dealer distributes a secret $s$, the shares sent to players in dispute with the dealer are defined to be zero. That is, instead of using a standard Shamir secret sharing, the dealer picks a random polynomial $f$ such that $f(0)=s$ and $f(i)=0$ for each $P_{i}$ in dispute with the dealer. Then the shares $f(i)$ are sent to each player $P_{i}$. The shares of the players in dispute with the dealer are called Kudzu shares. Since the set of players in dispute with the dealer is public knowledge, every player will know the shares sent to players in dispute with the dealer. This prevents the recipients from lying about the shares they received later in the protocol. The secret sharing scheme from [3] can also be adapted to implement Kudzu shares; ours is the first MPC protocol to implement Kudzu shares with MSP secret sharing for general adversaries.

One common technique used in verifiable secret sharing (VSS) protocols is double sharing, in which the dealer shares the secret and then shares the shares. This allows the players to reconstruct the secret in the presence of corrupt players. Double sharing was used in both [11] and [4]. Since the size of an MSP sharing is $d$, the size of a double sharing is (at least) $d^{2}$. So to achieve communication complexity linear in $d$, we use another method for reconstructing. Throughout the protocol, each sharing is a linear combination of sharings generated by (possibly) multiple players during different executions of the VSS share protocol. So when an inconsistency is found during reconstruction in the protocol LC-Reconstruct, the players engage in a process similar to the bisection method to locate a single sharing for which there is an inconsistency. Then using the authentication/verification tags generated when sharing that value, the players can determine who the corrupt player is.

\section{Definitions and Assumptions}

Our MPC protocol is designed for a synchronous network with secure point-to-point channels and an authenticated broadcast channel. The players are to compute an arithmetic circuit over a finite field $\mathbb{F}$ of size $|\mathbb{F}|=2^{\kappa}$. We let $c_{I}, c_{M}, c_{R}, c_{O}$ denote the number of input, multiplication, random, and output gates (respectively) in the circuit. The total size of the circuit is $\mathcal{C}=c_{I}+c_{M}+c_{R}+c_{O}$. The multiplicative depth of the circuit is $\mathcal{D}$.

We denote the player set by $\mathcal{P}$ and the adversary structure by $\mathcal{A} \subset 2^{\mathcal{P}}$. Adversary structures are monotone, meaning that if $A \in \mathcal{A}$, then any subset of $A$ is in $\mathcal{A}$. We denote by $\overline{\mathcal{A}}$ the set of maximal sets in $\mathcal{A}$ (i.e., the set of all sets in $\mathcal{A}$ that are not proper subsets of any other sets in $\mathcal{A}$ ). An adversary structure $\mathcal{A}$ is said to be $Q 2$ if no two sets in the adversary structure cover the entire player set; that is, $\mathcal{A}$ is $Q 2$ if $A, B \in \mathcal{A} \Rightarrow A \cup B \neq \mathcal{P}$. Note that for threshold adversaries, the requirement that $t<n / 2$ is one example 
of a $Q 2$ adversary structure. Our MPC protocol is able to tolerate an malicious, adaptive adversary whose corruption pattern is specified by a $Q 2$ adversary structure.

We denote by $\mathcal{D} i s p$ the set of pairs of players who are in dispute with one another. If at any time a dispute arises between player $P_{i}$ and player $P_{j}$, (i.e., one of them says that the other is lying), the pair $\left\{P_{i}, P_{j}\right\}$ is added to Disp. Since all disputes are handled over the broadcast channel, each player has the same record of which pairs of players are in $\mathcal{D} i s p$. We define $\mathcal{D} i s p_{i}=\left\{P_{j} \mid\left\{P_{j}, P_{i}\right\} \in \mathcal{D} i s p\right\}$. If at any time the set $\mathcal{D} i s p_{i}$ is no longer in $\mathcal{A}$, that means that at least one honest player has accused $P_{i}$, and therefore all players know that $P_{i}$ must be corrupt. We use the set $\mathcal{C}$ orr to denote the set of players known by all players to be corrupt. We define $\mathcal{G o o d}=\mathcal{P}-\mathcal{C}$ orr .

Most of the protocols in this paper use dispute control and will terminate when one or more pairs of players are added to Disp. In this case, the protocol terminates unsuccessfully. We handle unsuccessful termination of protocols as in [2]. Namely, the circuit is divided into (roughly) $n^{2}$ segments, and if one of the sub-protocols terminates unsuccessfully during the computation for a segment, that segment is started over from the beginning. A new dispute is found at each unsuccessful termination, and since there can be at most $n^{2}$ disputes, this does not affect the asymptotic complexity of the protocol. Throughout this paper, we will assume (without explicitly stating it) that if a sub-protocol invoked by a parent protocol terminates unsuccessfully, then the parent protocol terminates unsuccessfully.

Let $M$ be a matrix over $\mathbb{F}$ with $d$ rows and $e$ columns, and let $\mathbf{a}=(1,0,0, \cdots, 0)^{\top} \in \mathbb{F}^{e}$. The triple $(\mathbb{F}, M, \mathbf{a})$ is called a monotone span program $(\mathrm{MSP}) .{ }^{3}$ Define $\left(x_{1}, x_{2}, \ldots, x_{\ell}\right)^{\top} *\left(y_{1}, y_{2}, \ldots, y_{\ell}\right)^{\top}=$ $\left(x_{1} y_{1}, x_{2} y_{2}, \ldots, x_{\ell} y_{\ell}\right)^{\top}$, and suppose $\boldsymbol{\lambda}$ is a vector in $\mathbb{F}^{d}$. We call $(\mathbb{F}, M, \mathbf{a}, \boldsymbol{\lambda})$ a multiplicative $M S P$ if $(\mathbb{F}, M, \mathbf{a})$ is an MSP and if $\boldsymbol{\lambda}$ has the property that

$$
\left\langle\boldsymbol{\lambda}, M \mathbf{b} * M \mathbf{b}^{\prime}\right\rangle=\langle\mathbf{a}, \mathbf{b}\rangle \cdot\left\langle\mathbf{a}, \mathbf{b}^{\prime}\right\rangle
$$

for all $\mathbf{b}, \mathbf{b}^{\prime}$. In this case, $\boldsymbol{\lambda}$ is called the recombination vector.

Each row of $M$ will be labeled with an index $i(1 \leq i \leq n)$, so that each row corresponds to some player. For any nonempty subset $A \subset\{1,2, \ldots, n\}, M_{A}$ denotes the matrix consisting of all rows whose index is in $A$. For a given adversary structure $\mathcal{A}$, we say that the $\operatorname{MSP}(\mathbb{F}, M, \mathbf{a})$ represents $\mathcal{A}$ if

$$
A \notin \mathcal{A} \Longleftrightarrow \mathbf{a} \in \operatorname{Im} M_{A}^{\top} \text {. }
$$

It is shown in section 3.1 that the condition $\mathbf{a} \in \operatorname{Im} M_{A}^{\top}$ implies that a secret shared using $M$ can be reconstructed from the shares of players in $A$.

The size of the multiplicative MSP representing the adversary structure (measured as the number of rows in the matrix) is of prime importance in analyzing the communication complexity of the MPC protocol, because secrets are shared as a vector in the image of $M$. There is an algorithm in [3] that allows one to construct a multiplicative MSP from $\overline{\mathcal{A}}$ for any $Q 2$ adversary structure $\mathcal{A}$ such that the number of rows of $M$ is polynomially related to $|\overline{\mathcal{A}}|$.

A "basic" sharing of a value $w$ created using the MSP (as generated by the protocol Share below) is denoted by $[w]$. The share of player $P_{i}$ is denoted by $w_{i}$. Note that in general $w_{i}$ will be a vector, since it represents a portion of a vector in the image of $M$, although it could be a single-element vector. This secret sharing scheme is linear in that each player can compute a sharing of an affine combination of already-shared secrets by performing local computations.

\section{The Protocols}

This section describes the MPC protocol and all sub-protocols. Proofs are deferred to Appendix A.1.

\footnotetext{
${ }^{3}$ The definition of MSP in [3] allows a to be any fixed vector, but it is convenient to choose a as we have.
} 


\subsection{Secret Sharing}

Our MPC protocol uses a "basic" secret sharing protocol and constructs a verifiable secret sharing (VSS) protocol by combining the basic protocol with information checking [2]. The basic secret sharing protocolwhich is described in this section - is essentially the secret sharing protocol of [3], except that it is implemented with Kudzu shares [2]. We first review the secret sharing protocol of [3] and then prove that this can be implemented with Kudzu shares.

Given an MSP with matrix $M$ of size $d \times e$ as described in section 2, the secret sharing protocol of [3] proceeds as follows: The dealer with secret $s$ picks $e-1$ random values $r_{2}, \ldots, r_{e}$, constructing a vector $\mathbf{s}=\left(s, r_{2}, r_{3}, \ldots, r_{e}\right)$. The dealer then computes $\mathbf{b}=M \mathbf{s}=[s]$ and sends some of the entries of the vector b to each player. It is shown in [3] how to construct multiplicative MSPs suitable for secret sharing for any given $Q 2$ adversary structure.

To implement Kudzu shares with this secret sharing scheme, we note that the secret sharing scheme described above is perfectly private (proved in [3]). In other words, the adversary's view of the vector $\mathbf{b}$ is independent of the secret being shared. So for a sharing $\mathbf{b}=[s]$ and a set $A \in \mathcal{A}$, the dealer can construct a sharing of zero [0] such that $A$ 's view of [0] is the same as $A$ 's view of $[s]$. Then the sharing $[s]-[0]$ is a sharing of $s$ with Kudzu shares, as the shares of all players in dispute with the dealer will be zero.

Protocol: Share $(D, s)$

The dealer $P_{D}$ wants to share a secret $s \in \mathbb{F}$. He selects random values $r_{2}, r_{3}, \ldots, r_{e} \in \mathbb{F}$, constructing a vector $\mathbf{s}=\left(s, r_{2}, r_{3}, \ldots, r_{e}\right) \in \mathbb{F}^{e}$. The random values $r_{2}, r_{3}, \ldots, r_{e}$ are chosen subject to the constraint that the shares of players in dispute with $P_{D}$ must be all-zero vectors. The dealer then computes $[s]=\mathbf{b}=M \mathbf{s}$, where $M$ is the MSP corresponding to $\mathcal{A}$. The dealer sends $b_{j}=M_{j}$ s to each $P_{j} \notin \mathcal{D} i s p_{D}$ (where $b_{j}$ is the vector of components of $\mathbf{b}$ corresponding to player $P_{j}$ ).

For a value $v \in \mathbb{F}$, we call the canonical sharing of $v$ the sharing for which $r_{2}, r_{3}, \ldots, r_{e}$ are all zero.

In this paper, we will represent the complexity of each protocol in a table. The columns denote communication bandwidth, broadcast bandwidth, communication rounds, and broadcast rounds (abbreviated $\mathrm{CB}, \mathrm{BCB}, \mathrm{CR}$, and $\mathrm{BCR}$, respectively). The two rows represent the complexity in the absence of a dispute and the added complexity per dispute. It is assumed that the communication and broadcast bandwidths are stated asymptotically (i.e., the big-O is not written, but is assumed). Bandwidth is measured in field elements, so one would have to multiply by $\kappa$ to compute the bandwidth in bits.

\begin{tabular}{|l|cccc|}
\hline Share & $C B$ & $B C B$ & $C R$ & $B C R$ \\
\hline WithoutDispute & $d$ & 0 & 1 & 0 \\
PerDispute & 0 & 0 & 0 & 0 \\
\hline
\end{tabular}

Lemma 1. The protocol Share is a secret sharing scheme secure against any malicious, adaptive adversary with $Q 2$ adversary structure $\mathcal{A}$.

We now show how reconstruction is performed on a sharing. Suppose we want to reconstruct a secret using the shares of some set $A$ of players satisfying $A \notin \mathcal{A}$. Since the MSP represents $\mathcal{A}$, by definition this means that $\mathbf{a} \in \operatorname{Im} M_{A}^{\top}$. So there is some vector $\boldsymbol{\omega}_{A}$ satisfying $M_{A}^{\top} \boldsymbol{\omega}_{A}=\mathbf{a}$. If $[w]_{A}$ represents the shares of $[w]$ held by players in $A$ and $\mathbf{s}=\left(w, r_{2}, \ldots, r_{e}\right)^{\top}$ represents the vector used in Share to generate the sharing $[w]$, then we can reconstruct the secret as

$$
\left\langle\boldsymbol{\omega}_{A},[w]_{A}\right\rangle=\left\langle\boldsymbol{\omega}_{A}, M_{A} \mathbf{s}\right\rangle=\left\langle M_{A}^{\top} \boldsymbol{\omega}_{A}, \mathbf{s}\right\rangle=\langle\mathbf{a}, \mathbf{s}\rangle=w .
$$




\subsection{Information Checking}

Information checking (IC) is a scheme by which a sender can give a message to a receiver along with some auxiliary information (verification tags); the sender also gives some auxiliary information (authentication tags) to a verifier. This is done such that at a later time, if there is a disagreement about what the sender gave the receiver, the verifier can act as an "objective third party" to settle the dispute. We ensure that the verifier does not find out any information about the message (until a dispute arises).

The protocols Distribute-Tags and Check-Message are are variants of those used in [2], so their explicit description is deferred to Appendix A.2. The main difference is that we use an extension field $\mathbb{G}$ of $\mathbb{F}$ to allow the sender to produce tags for messages of length at most $d$. Since $d$ can be as much as exponential in $n$, this is a much larger message size than that allowed in [2].

Lemma 2. The following four facts hold.

1. If Distribute-Tags succeeds and $P_{V}, P_{R}$ are honest, then with overwhelming probability $P_{V}$ accepts the linear combination of the messages in Check-Message.

2. If Distribute-Tags fails, then a new pair of players is added to Disp, and at least one of the two players is corrupt.

3. If $P_{S}$ and $P_{V}$ are honest, then with overwhelming probability, $P_{V}$ rejects any fake message $\mathbf{m}^{\prime} \neq \mathbf{m}$ in Check-Message.

4. If $P_{S}$ and $P_{R}$ are honest, then $P_{V}$ obtains no information about $\mathbf{m}$ in Distribute-Tags (even if it fails).

The proof of this lemma and the complexities of the information checking protocols are given in Appendix A.2.

\subsection{Verifiable Secret Sharing}

A verifiable secret sharing (VSS) scheme consists of two protocols, VSS and VSS-Reconstruct. We use the following definition of secret sharing:

Definition 1. Consider a protocol VSS for distributing shares of a secret $s$ and a protocol VSS-Reconstruct for reconstructing s from the shares. We call this pair of protocols a VSS scheme if the following properties are satisfied (with overwhelming probability):

1. Termination: Either all honest players complete VSS, or a new dispute is found. All honest players will complete VSS-Reconstruct.

2. Privacy: If the dealer is honest, then before the beginning of VSS-Reconstruct, the adversary has no information on the shared secret $s$.

3. Correctness: Once all honest players complete VSS there is a fixed value $r$ such that:

3.1 If the dealer was honest throughout VSS, then $r=s$.

3.2 Whether or not the dealer is honest, at the end of VSS-Reconstruct the honest players will reconstruct $r$.

The following protocol allows a dealer $P_{D} \in \mathcal{G o o d}$ to verifiably share $\ell$ values. To verify correctness, each player acts as verifier and requests a random linear combination of these sharings (masked by a random sharing) to be opened. If the sharing is inconsistent (meaning that it is not in the span of $M$ ), then dispute resolution occurs. When $P_{D}$ shares secrets, he utilizes information checking to produce authentication and verification tags in case a disagreement occurs later as to what was sent.

Protocol: $\operatorname{VSS}\left(P_{D}, \ell, s^{(1)}, \ldots, s^{(\ell)}\right)$ 
We assume that $P_{D} \in \mathcal{G}$ ood wants to share $s^{(1)}, \ldots, s^{(\ell)}$. If $P_{D} \in \mathcal{C}$ orr, then all the sharings will be defined to be all-zero sharings.

\section{Distribution}

1.1 $P_{D}$ selects $n$ extra random values $u^{(1)}, \ldots, u^{(n)}$, and then invokes Share to share $\left\{u^{(i)}\right\}_{i=1}^{n}$ and $\left\{s^{(i)}\right\}_{i=1}^{\ell}$.

1.2 For each pair $P_{R}, P_{V} \notin \mathcal{D} i s p_{D}$ such that $\left\{P_{R}, P_{V}\right\} \notin \mathcal{D} i s p$, invoke Distribute-Tags $\left(P_{D}, P_{R}, P_{V}, \mathbf{s}_{R}\right)$, where

$$
\mathbf{s}_{R}=\left(s_{R}^{(1)}, \ldots, s_{R}^{(\ell)}, u_{R}^{(1)}, \ldots, u_{R}^{(n)}\right)
$$

(remember that each $s_{R}^{(k)}$ and $u_{R}^{(k)}$ is a vector).

2. Verification

The following steps are performed in parallel for each $P_{V} \notin \mathcal{D} i s p_{D}$, who acts as verifier.

2.1 $P_{V}$ choses a random vector $\left(r_{1}, \ldots, r_{\ell}\right) \in \mathbb{F}^{\ell}$ and broadcasts it.

2.2 Each player $P_{i} \notin \mathcal{D} i s p_{D}$ sends his share of $\sum_{k=1}^{\ell} r_{k}\left[s^{(k)}\right]+\left[u^{(V)}\right]$ to $P_{V}$.

2.3 If $P_{V}$ finds that the shares he received in the previous step (together with the Kudzu shares) form a consistent sharing, (i.e., it is a vector in the span of $M_{\mathcal{P}-\mathcal{C} o r r}$ ), then $P_{V}$ broadcasts (accept, $\left.P_{D}\right)$, and the protocol terminates. Otherwise, $P_{V}$ broadcasts (reject, $P_{D}$ ).

3. Fault Localization

For the lowest $P_{V}$ that broadcast "(reject, $\left.P_{D}\right)$ " in the previous step, then the following steps are performed.

3.1 $P_{D}$ broadcasts each share of $\sum_{k=1}^{\ell} r_{k}\left[s^{(k)}\right]+\left[u^{(V)}\right]$. If this sharing is inconsistent, then $P_{D}$ is added to $\mathcal{C}$ orr and the protocol terminates.

3.2 If the protocol did not terminate in the last step, then there is a share of some player $P_{i} \notin \mathcal{D} i s p_{D}$ that broadcast a different share than $P_{D}$. So $P_{V}$ broadcasts (accuse, $P_{i}, P_{D}, v_{i}, v_{D}$ ), where $v_{i}$ is the value of the share sent by $P_{i}$ and $v_{D}$ the value sent by $P_{D}$.

3.3 If $P_{i}$ disagrees with the value $v_{i}$ broadcast by $P_{V}$, then $P_{i}$ broadcasts (dispute, $P_{i}, P_{V}$ ), the set $\left\{P_{i}, P_{V}\right\}$ is added to $\mathcal{D} i s p$, and the protocol terminates.

3.4 If $P_{D}$ disagrees with the value $v_{D}$ broadcast by $P_{V}$, then $P_{D}$ broadcasts (dispute, $P_{D}, P_{V}$ ), the set $\left\{P_{D}, P_{V}\right\}$ is added to $\mathcal{D} i s p$, and the protocol terminates.

3.5 If neither $P_{i}$ nor $P_{D}$ complained in the previous two steps, then $\left\{P_{i}, P_{D}\right\}$ is added to $\mathcal{D} i s p$, and the protocol terminates.

\begin{tabular}{|l|cccc|}
\hline VSS & $C B$ & $B C B$ & $C R$ & $B C R$ \\
\hline WithoutDispute & $\ell d+n d+n^{2} \kappa \log d$ & $n \ell+n^{2}$ & 4 & 3 \\
PerDispute & 0 & $d$ & 0 & 4 \\
\hline
\end{tabular}

Note that this protocol can be easily modified to (verifiably) construct multiple sharings of $1 \in \mathbb{F}$, (i.e., the multiplicative identity). We simply require that all $s^{(k)}=1$ for all $k=1, \ldots, \ell$ and $u^{(k)}=1$ for all $k=1, \ldots, n$, and in step 2.3, $P_{V}$ checks not only that the sharing is consistent, but that it is a sharing of $\sum_{k=1}^{\ell} r_{k}+1$; step 3.1 is similarly altered. Furthermore, in the fault localization section, the players check not only that sharings are consistent, but that they are sharings of the correct values. We refer to this modified protocol by VSS-One.

Lemma 3. The protocol VSS is statistically correct and perfectly private. More explicitly:

1. If VSS terminates successfully:

1.1 With overwhelming probability, the values $s^{(1)}, \ldots, s^{(\ell)}$ are correctly shared. 
1.2 With overwhelming probability, for each ordered triple of players $\left(P_{i}, P_{j}, P_{k}\right)$ that are not in dispute with one another, ${ }^{4} P_{k}$ has correct authentication tags for the shares sent from $P_{i}$ to $P_{j}$.

2. If the protocol terminates with a dispute, then the dispute is new.

3. Regardless of how the protocol terminates, the adversary gains no information on the $s^{(1)}, \ldots, s^{(\ell)}$ shared by honest players.

The protocol VSS-Reconstruct, is used to reconstruct a sharing generated by a single player. The reconstruction protocol used in the main MPC protocol (called LC-Reconstruct) will be used to reconstruct linear combinations of sharings that were shared by multiple dealers. Since VSS-Reconstruct is largely the same as the reconstruction protocol in [2], using the authentication and verification tags generated in VSS-Share, it is deferred to Appendix A.3.

Lemma 4. The pair VSS and VSS-Reconstruct described above constitute a VSS scheme.

\subsection{Reconstructing Linear Combinations of Sharings}

The following protocol is used to reconstruct linear combinations of sharings of secrets that have been shared using VSS. It assumes that each sharing $[w]$ is a sum of sharings $\left[w^{(1)}\right]+\cdots+\left[w^{(n)}\right]$, where $\left[w^{(i)}\right]$ is a linear combination of sharings shared by player $P_{i}$. Note that the protocol has some chance of failure. However, whenever the protocol fails, a new player is added to $\mathcal{C}$ orr, so it can fail only $O(n)$ times in the entire MPC protocol.

The technique for using the authentication tags in LC-Reconstruct is non-standard, and deserves a bit of explanation. If the initial broadcasting of shares of $[w]$ is inconsistent, then the players open each $\left[w^{(j)}\right]$. If $\left[w^{(j)}\right]$ is the first such sharing that is inconsistent, then the players will want to use the authentication tags to determine who is lying. However, $\left[w^{(j)}\right]$ is a linear combination of sharings that were generated with VSS. Each of these initial sharings has authentication tags, but there is no means for combining the tags to get tags for $\left[w^{(j)}\right]$.

So the players need to localize which of the sharings in the linear combination $\left[w^{(j)}\right]=a_{1}\left[s^{(1)}\right]+\cdots+$ $a_{m}\left[s^{(m)}\right]$ is inconsistent. One way to do this would be to have $P_{j}$ state which player he accuses of lying and have that player broadcast shares of each $\left[s^{(k)}\right]$ (or if $P_{j}$ is corrupt, all players broadcast their shares of each $\left.\left[s^{(k)}\right]\right)$. Once this is done, the players could use the tags for whichever share $P_{j}$ claims is corrupt to determine who was lying. Although this approach would work, it would result in an enormous communication complexity. Therefore, instead of opening all of the $\left[s^{(k)}\right]$ all at once, the players use a "divide-and-conquer" technique: Break the sum into two halves, determine which sum has the inconsistency, break that sum in half, and so on until the players reach an individual sharing, at which point they can use the authentication tags.

\section{Protocol: LC-Reconstruct $([w])$}

Throughout this protocol, if a player ever refuses to send or broadcast something that the protocol requires, that player is added to $\mathcal{C}$ orr, and the protocol terminates.

1. Each $P_{i} \notin \mathcal{C}$ orr broadcasts his share $w_{i}$ of $[w]$.

2. If the sharing broadcast in the previous step is consistent, then the players reconstruct $w$ as described in section 3.1, and the protocol terminates.

3. If the sharing was inconsistent, each $P_{i} \notin \mathcal{C}$ orr broadcasts his share $w_{i}^{(j)}$ for each $P_{j} \in \mathcal{P}$.

4. If any player $P_{i}$ broadcasted values such that his summands do not match his sum (i.e., if $w_{i} \neq \sum_{j=1}^{n} w_{i}^{(j)}$ ), then all such players are added to $\mathcal{C}$ orr, and the protocol terminates.

\footnotetext{
${ }^{4}$ That is, no pair of players in the triple are in dispute with each other.
} 
5. For the lowest $j$ such that the shares of $w^{(j)}$ broadcast in step 3 are inconsistent, one of two steps is performed: If $P_{j} \notin \mathcal{C}$ orr proceed to step 6 . Otherwise, proceed to step 7.

6. $P_{j} \notin \mathcal{C}$ orr

6.1 $P_{j}$ broadcasts (accuse, $i$ ) for the player $P_{i}$ he believes to have sent an incorrect share.

6.2 Since $\left[w^{(j)}\right]$ is a linear combination of sharings dealt by $P_{j}$, the players (internally) think of $\left[w^{(j)}\right]$ as $a_{1}\left[s^{(1)}\right]+\cdots+a_{m}\left[s^{(m)}\right]$, where each $\left[s^{(k)}\right]$ was generated with VSS and each $a_{k}$ is non-zero. We arrange the $s^{(k)}$ 's according to the order in which they were dealt.

6.3 From the sharings $a_{1}\left[s^{(1)}\right], \ldots, a_{m}\left[s^{(m)}\right]$, define two sharings $a_{1}\left[s^{(1)}\right]+\cdots+a_{\lfloor m / 2\rfloor}\left[s^{(\lfloor m / 2\rfloor)}\right]$ and $a_{\lfloor m / 2\rfloor+1}\left[s^{(\lfloor m / 2\rfloor+1)}\right]+\cdots+a_{m}\left[s^{(m)}\right]$. The player $P_{i}$ accused in step 6.1 broadcasts his share of each of these two sharings.

6.4 If $P_{i}$ broadcast shares of summands in the previous step that do not match up with the previously sent share of their sum, then $P_{i}$ is added to $\mathcal{C}$ orr, and the protocol terminates.

6.5 Player $P_{j}$ broadcasts which of the sharings broadcast in step 6.3 he disagrees with. If this is a single sharing $a_{k}\left[s^{(k)}\right]$, then the players proceed to step 6.6. Otherwise, if the sharing is some sum $a_{k_{1}}\left[s^{\left(k_{1}\right)}\right]+\cdots+a_{k_{2}}\left[s^{\left(k_{2}\right)}\right]$, then the players return to step 6.3 , but with $a_{1}\left[s^{(1)}\right], \ldots, a_{m}\left[s^{(m)}\right]$ replaced by $a_{k_{1}}\left[s^{\left(k_{1}\right)}\right]+\cdots+a_{k_{2}}\left[s^{\left(k_{2}\right)}\right]$.

6.6 At this point, $P_{i}$ has broadcast his share of $a_{k}\left[s^{(k)}\right]$, and $P_{j}$ has broadcast that he disagrees with this share. For each $P_{V} \notin \mathcal{D} i s p_{j} \cup \mathcal{D} i s p_{i}$, the players invoke Check-Message $\left(P_{i}, P_{V}, \mathbf{s}_{i}\right)$, where $\mathbf{s}_{i}$ is the vector defined in step 1.2 of the invocation of VSS in which $\left[s^{(k)}\right]$ was shared.

6.7 If $P_{i}$ sent shares to $P_{V}$ in the invocation of Check-Message that do not match with the share of $a_{k}\left[s^{(k)}\right]$, then $P_{V}$ broadcasts (accuse, $i$ ), and $\left\{P_{i}, P_{V}\right\}$ is added to $\mathcal{D} i s p$.

6.8 For each $P_{V} \notin \mathcal{D} i s p_{i}$ that rejected the message sent by $P_{i}$ in the invocation of Check-Message, $\left\{P_{i}, P_{V}\right\}$ is added to $\mathcal{D} i s p$. For each $P_{V}$ that accepted the message, $\left\{P_{j}, P_{V}\right\}$ is added to Disp.

6.9 At this point, all players are in dispute with either $P_{i}$ or $P_{j}$. By the $Q 2$ property of the adversary structure $\mathcal{A}$, this means that one of $\mathcal{D} i s p_{i}$ or $\mathcal{D} i s p_{j}$ is no longer in $\mathcal{A}$. If $\mathcal{D} i s p_{i} \notin \mathcal{A}$ then $P_{i}$ is added to $\mathcal{C}$ orr , and if $\mathcal{D} i s p_{j} \notin \mathcal{A}$, then $P_{j}$ is added to $\mathcal{C}$ orr. Then the protocol terminates.

7. $P_{j} \in \mathcal{C}$ orr

7.1 Since $\left[w^{(j)}\right]$ is a linear combination of sharings dealt by $P_{j}$, the players (internally) think of $\left[w^{(j)}\right]$ as $a_{1}\left[s^{(1)}\right]+\cdots+a_{m}\left[s^{(m)}\right]$, where each $\left[s^{(k)}\right]$ was generated with VSS and each $a_{k}$ is non-zero. We arrange the $s^{(k)}$ 's according to the order in which they were dealt.

7.2 From the sharings $a_{1}\left[s^{(1)}\right], \ldots, a_{m}\left[s^{(m)}\right]$, define two sharings $a_{1}\left[s^{(1)}\right]+\cdots+a_{\lfloor m / 2\rfloor}\left[s^{(\lfloor m / 2\rfloor)}\right]$ and $a_{\lfloor m / 2\rfloor+1}\left[s^{(\lfloor m / 2\rfloor+1)}\right]+\cdots+a_{m}\left[s^{(m)}\right]$. Each player not in $\mathcal{C}$ orr broadcasts his share of each of these two sharings.

7.3 Any player who broadcast shares of summands in the previous step that do not match up with the previously sent share of their sum is added to $\mathcal{C}$ orr, and the protocol terminates.

7.4 If the players reach this step, then one of the sharings broadcast in step 7.2 is inconsistent. If this is a single sharing $a_{k}\left[s^{(k)}\right]$, then the players proceed to step 7.5. Otherwise, if the sharing is some sum $a_{k_{1}}\left[s^{\left(k_{1}\right)}\right]+\cdots+a_{k_{2}}\left[s^{\left(k_{2}\right)}\right]$, then the players return to step 7.2 , but with $a_{1}\left[s^{(1)}\right], \ldots, a_{m}\left[s^{(m)}\right]$ replaced by $a_{k_{1}}\left[s^{\left(k_{1}\right)}\right]+\cdots+a_{k_{2}}\left[s^{\left(k_{2}\right)}\right]$.

7.5 The players invoke VSS-Reconstruct for the sharing $\left[s^{(k)}\right]$ decided upon in the last execution of step 7.4 (however, they skip step 1 of VSS-Reconstruct, since shares of $a_{k}\left[s^{(k)}\right]$ have already been broadcast).

7.6 The invocation of VSS-Reconstruct in the previous step will have added a new player to $\mathcal{C}$ orr, so the protocol terminates. 


\begin{tabular}{|l|cccc|}
\hline LC-Reconstruct & $C B$ & $B C B$ & $C R$ & $B C R$ \\
\hline WithoutDispute & 0 & $d$ & 0 & 1 \\
PerDispute & $n^{2} \ell+n^{2} \kappa \log d$ & $n^{2}+n d+d \log \mathcal{C}$ & 2 & $6+\log \mathcal{C}$ \\
\hline
\end{tabular}

Lemma 5. If $[w]$ is a linear combination of sharings generated with VSS, then with overwhelming probability, an invocation of LC-Reconstruct $([w])$ will either reconstruct the correct value $w$ or add a new player to Corr. Furthermore, LC-Reconstruct does not leak any information about any sharing other than $[w]$ to the adversary.

\subsection{Generating Random Values}

The following protocol allows the players to generate a publicly known random vector $\left(s^{(1)}, \ldots, s^{(\ell)}\right)$. If the protocol fails (which occurs if one of its sub-protocols fails), then a new dispute pair is found.

Protocol: Generate-Randomness $(\ell)$

1. Every player $P_{i} \notin \mathcal{C}$ orr selects a random summand vector $s^{(1, i)}, \ldots, s^{(\ell, i)}$.

2. Call VSS $\left(P_{i}, \ell, s^{(1, i)}, \ldots, s^{(\ell, i)}\right)$ to let every $P_{i} \notin \mathcal{C}$ orr verifiably share his summand vector.

3. Call LC-Reconstruct $\ell$ times in parallel to reconstruct the sum sharings $s^{(1)}=\sum_{P_{i} \notin \mathcal{C} o r r} s^{(1, i)}, \ldots, s^{(\ell)}=$ $\sum_{P_{i} \notin \mathcal{C} \text { orr }} s^{(\ell, i)}$.

\begin{tabular}{|l|cccc|}
\hline Generate-Randomness & $C B$ & $B C B$ & $C R$ & $B C R$ \\
\hline WithoutDispute & $n \ell d+n^{2} d+n^{3} \kappa \log d$ & $n^{2} \ell+n^{3}+d \ell$ & 4 & 4 \\
PerDispute & $n^{2} \ell+n^{2} \kappa \log d$ & $n^{2}+n d+d \log \mathcal{C}$ & 2 & $6+\log \mathcal{C}$ \\
\hline
\end{tabular}

Lemma 6. If Generate-Randomness terminates successfully, then the reconstructed vector is random. If Generate-Randomness terminates unsuccessfully, then a new dispute is found.

\subsection{Generating Multiplication Triples}

The following protocol allows the players to verifiably generate random sharings of triples $(a, b, c)$ such that $a b=c$. The idea is that a random $a^{(k)}$ is generated, and then each $P_{i}$ is "responsible for" creating a random triple $a^{(k)} b^{(i, k)}=c^{(i, k)}$. To verify correctness, the $P_{i}$ also creates a triple $a^{(k)} \widetilde{b}^{(i, k)}=\widetilde{c}^{(i, k)}$, and this is used to mask an opening of $a^{(k)} b^{(i, k)}-c^{(i, k)}$. Once all these triples are checked, the final triple is defined to be $\left(a^{(k)}, \sum_{i=1}^{n} b^{(i, k)}, \sum_{i=1}^{n} c^{(i, k)}\right)$.

\section{Protocol: Multiplication-Triple $(\ell)$}

1. Generating Triples

1.1 Each $P_{i} \notin \mathcal{C}$ orr invokes VSS $\left(P_{i}, 2 \ell n+3 \ell\right)$ to generate uniformly random sharings and VSS-One $\left(P_{i}, 2 \ell n\right)$ to generate sharings of $1 \in \mathbb{F}$; these invocations are done in parallel. Denote the random sharings of player $P_{i}$ by $\left(\left[a^{(i, 1)}\right], \ldots,\left[a^{(i, \ell)}\right]\right),\left(\left[b^{(i, 1)}\right], \ldots,\left[b^{(i, \ell)}\right]\right),\left(\left[\widetilde{b}^{(i, 1)}\right], \ldots,\left[\widetilde{b}^{(i, \ell)}\right]\right)$, and $\left\{\left(\left[r^{(i, j, 1)}\right], \ldots,\left[r^{(i, j, \ell)}\right]\right)\right.$, $\left.\left(\left[\widetilde{r}^{(i, j, 1)}\right], \ldots,\left[\widetilde{r}^{(i, j, \ell)}\right]\right)\right\}_{j=1}^{n}$ and the sharings of ones by $\left\{\left(\left[1^{(i, j, 1)}\right], \ldots,\left[1^{(i, j, \ell)}\right]\right),\left(\left[\widetilde{1}^{(i, j, 1)}\right], \ldots,\left[\widetilde{1}^{(i, j, \ell)}\right]\right)\right\}_{j=1}^{n}$. The sharings of players in $\mathcal{C}$ orr are defined to be all-zero sharings. 
1.2 For each $k=1, \ldots, \ell$ and each $i$ such that $P_{i} \notin \mathcal{C}$ orr , the players define and locally compute

$$
\begin{gathered}
{\left[a^{(k)}\right]=\sum_{m=1}^{n}\left[a^{(m, k)}\right]} \\
{\left[r^{(i, k)}\right]=\sum_{m=1}^{n}\left[r^{(i, m, k)}\right]} \\
{\left[1^{(i, k)}\right]=\sum_{m=1}^{n}\left[1^{(m, i, k)}\right]+w\left[1^{(i, i, k)}\right],}
\end{gathered}
$$

where $w \in \mathbb{F}$ is the unique element that makes $\left[1^{(i, k)}\right]$ a sharing of 1 . The sharings $\left[\widetilde{r}^{(i, k)}\right]$ and $\left[\widetilde{1}^{(i, k)}\right]$ are similarly defined.

1.3 Each $P_{j} \notin \mathcal{C}$ orr sends his share of $\left[a^{(k)}\right]\left[b^{(i, k)}\right]+\left[r^{(i, k)}\right]\left[1^{(i, k)}\right]$ and $\left[a^{(k)}\right]\left[\widetilde{b}^{(i, k)}\right]+\left[\widetilde{r}^{(i, k)}\right]\left[\widetilde{1}^{(i, k)}\right]$ to $P_{i} \notin \mathcal{C}$ orr for each $k=1, \ldots, \ell$. (The shares of players in $\mathcal{C}$ orr will be Kudzu shares, so $P_{i}$ knows those shares as well.)

1.4 Each $P_{i} \notin \mathcal{C}$ orr applies the recombination vector $\lambda$ to the shares of $D^{(i, k)}=a^{(k)} b^{(i, k)}+r^{(i, k)}$ and $\widetilde{D}^{(i, k)}=a^{(k)} \widetilde{b}^{(i, k)}+\widetilde{r}^{(i, k)}$ received in the previous step to compute $D^{(i, k)}$ and $\widetilde{D}^{(i, k)}$ for each $k=1, \ldots, \ell$.

1.5 Each $P_{i}$ broadcasts $D^{(i, k)}$ and $\widetilde{D}^{(i, k)}$ for each $k=1, \ldots, \ell$.

1.6 Each player locally computes $\left[c^{(i, k)}\right]=D^{(i, k)}-\left[r^{(i, k)}\right]$ and $\left[\widetilde{c}^{(i, k)}\right]=\widetilde{D}^{(i, k)}-\left[\widetilde{r}^{(i, k)}\right]$ (using the canonical sharings of $D^{(i, k)}$ and $\widetilde{D}^{(i, k)}$ as defined in section 3.1).

\section{Error Detection}

2.1 The players invoke Generate-Randomness $(\ell)$ to generate a random vector $\left(s^{(1)}, \ldots, s^{(\ell)}\right)$.

2.2 Each player not in $\mathcal{D} i s p_{i}$ broadcasts his share of $\left[\widehat{b}^{(i, k)}\right]=\left[\widetilde{b}^{(i, k)}\right]+s^{(i)}\left[b^{(i, k)}\right]$ for each $i=1, \ldots, n$ and each $k=1, \ldots, \ell$.

2.3 If the sharing of some $\left[\widehat{b}^{(i, k)}\right]$ broadcast in the previous step is inconsistent, $P_{i}$ broadcasts (accuse, $P_{j}$ ) for some $P_{j} \notin \mathcal{D} i s p_{i}$ who broadcasted an incorrect share, then $\left\{P_{i}, P_{j}\right\}$ is added to $\mathcal{D} i s p$ and the protocol terminates.

2.4 The players invoke multiple instances of LC-Reconstruct in parallel to reconstruct $z^{(i, k)}=\left[a^{(k)}\right] \widehat{b}^{(i, k)}-$ $\left[\widetilde{c}^{(i, k)}\right]-s^{(k)}\left[c^{(i, k)}\right]$ for each $i=1, \ldots, n$ and each $k=1, \ldots, \ell$.

2.5 If all the $z^{(i, k)}$ reconstructed in the previous step are zero, then we define

$$
\begin{aligned}
& {\left[b^{(k)}\right]=\sum_{m=1}^{n}\left[b^{(m, k)}\right]} \\
& {\left[c^{(k)}\right]=\sum_{m=1}^{n}\left[c^{(m, k)}\right],}
\end{aligned}
$$

and the protocol terminates successfully with the multiplication triples taken to be $\left(a^{(k)}, b^{(k)}, c^{(k)}\right)$ for $k=1, \ldots, \ell$.

3. Fault Localization

If any $z^{(i, k)}$ reconstructed in step 2.4 is not zero, the following is done for the lexicographically lowest pair $(i, k)$ such that $z^{(i, k)} \neq 0$.

3.1 Each $P_{j}$ broadcasts his share of $\left[a^{(m, k)}\right],\left[\widetilde{r}^{(m, i, k)}\right]$, and $\left[r^{(m, i, k)}\right]$ for each $P_{m} \notin P_{j}$.

3.2 If $P_{i}$ sees that the shares of some $P_{j} \notin \mathcal{D} i s p_{i}$ sent in the previous step are inconsistent with the share sent in step 1.3 or 2.4 , then $P_{i}$ broadcasts (accuse, $P_{j}$ ); then $\left\{P_{j}, P_{i}\right\}$ is added to Disp and the protocol terminates.

3.3 Each $P_{m}$ examines the shares broadcast in the previous step of all sharings that $P_{m}$ generated. If $P_{m}$ notices that some $P_{j} \notin \mathcal{D} i s p_{m}$ broadcast an incorrect share in the previous step, then $P_{m}$ broadcasts (accuse, $P_{j}$ ); then $\left\{P_{m}, P_{j}\right\}$ is added to $\mathcal{D} i s p$ and the protocol terminates.

3.4 If no $P_{m}$ broadcast an accusation in the previous step, then $P_{i}$ is added to $\mathcal{C}$ orr and the protocol terminates. 


\begin{tabular}{|l|cccc|}
\hline Multiplication-Triple & $C B$ & $B C B$ & $C R$ & $B C R$ \\
\hline WithoutDispute & $n^{2} \ell d+n^{3} \kappa \log d$ & $n^{3} \ell+n \ell d$ & 9 & 11 \\
PerDispute & $n^{2} \ell+n^{2} \kappa \log d$ & $n^{2}+n d+d \log \mathcal{C}$ & 2 & $6+\log \mathcal{C}$ \\
\hline
\end{tabular}

Lemma 7. If Multiplication-Triple terminates unsuccessfully, then a new dispute is localized. If MultiplicationTriple succeeds, then it maintains statistical correctness and perfect privacy. That is, with overwhelming probability, at the end of the protocol the players hold sharings of $\ell$ multiplication triples $(a, b, c)$ with $c=a b$; in addition, the adversary has no information on $a, b$, or $c$ (other than that $c=a b$ ).

\subsection{Preparation Phase}

The following protocol prepares the circuit for computation by generating the required sharings. The protocol generates multiplication triples for the multiplication gates and random sharings for random gates. The task is broken into $n^{2}$ segments. The number of multiplication triples and random sharings generated in each segment are denoted by $L_{M}$ and $L_{R}$ (respectively), and we require $L_{M} \leq\left\lceil c_{M} / n^{2}\right\rceil$ and $L_{R} \leq\left\lceil\left(c_{R}\right) / n^{2}\right\rceil$.

\section{Protocol: Preparation-Phase}

Initialize $\operatorname{Corr}$ and $\mathcal{D}$ isp to the empty set. For each segment handling $L_{M}$ multiplication gates and $L_{R}$ random gates, the following steps are performed. If any of the subprotocols fails, then the segment is repeated.

1. Invoke Multiplication-Triple $\left(L_{M}\right)$. Assign one multiplication triple to each multiplication gate in this segment.

2. Each $P_{i} \notin \mathcal{C}$ orr invokes $\operatorname{VSS}\left(P_{i}, L_{R}, r^{(1, i)}, \ldots, r^{\left(L_{R}, i\right)}\right)$, sharing uniformly random values. (The sharings of corrupt players are defined to be all-zero sharings.)

3. We define $L_{R}$ random sharings by $\left[r^{(k)}\right]=\sum_{i=1}^{n}\left[r^{(k, i)}\right]$ for each $k=1, \ldots, L_{R}$. Assign one random sharing to each random gate in this segment.

\begin{tabular}{|l|cccc|}
\hline Preparation-Phase & $C B$ & $B C B$ & $C R$ & $B C R$ \\
\hline WithoutDispute & $n^{2}\left(c_{M}+c_{R}\right) d+n^{5} \kappa \log d$ & $n^{3}\left(c_{M}+c_{R}\right)+n\left(c_{M}+c_{R}\right) d$ & $13 n^{2}$ & $14 n^{2}$ \\
PerDispute & $\left(c_{M}+c_{R}\right)+n^{2} \kappa \log d$ & $n^{2}+n d+d \log \mathcal{C}$ & 2 & $6+\log \mathcal{C}$ \\
\hline
\end{tabular}

\subsection{Input Phase}

The goal of the input phase is to allow each player to share their inputs. We denote the number of inputs in a given segment by $L$. We require $L \leq\left\lceil c_{I} / n^{2}\right\rceil$, and we also require that each segment contain inputs from only one player.

\section{Protocol: Input-Phase}

For each segment, the following steps are executed to let the dealer $P_{D} \notin \mathcal{C}$ orr verifiably share $L$ inputs $s^{(1)}, \ldots, s^{(L)}$. If some invocation of VSS fails, then the segment fails and is repeated.

1. Each $P_{i} \notin \mathcal{C}$ orr invokes $\operatorname{VSS}\left(P_{i}, L, r^{(1, i)}, \ldots, r^{(L, i)}\right)$, sharing uniformly random values. (The sharings of corrupt players are defined to be all-zero sharings.) 
2. We define $L$ random sharings by $\left[r^{(k)}\right]=\sum_{i=1}^{n}\left[r^{(k, i)}\right]$ for each $k=1, \ldots, L$. Assign one random sharing to each input gate in this segment.

3. Each $P_{i} \notin \mathcal{D} i s p_{D}$ sends his share of each $\left[r^{(k)}\right]$ to $P_{D}$.

4. If $P_{D}$ finds that one of the sharings was inconsistent, he broadcasts the index of this sharing, and the following steps are performed. If they are all consistent, then the players proceed to step 5 .

4.1 If $P_{D}$ indicated that the random sharing $[r]$ was inconsistent, then each $P_{i} \notin \mathcal{D} i s p_{D}$ broadcasts their share of $[r]$.

4.2 If $P_{D}$ sees that some $P_{i}$ broadcast a different share than was sent privately, then $P_{D}$ broadcasts (accuse, $i),\left\{P_{D}, P_{i}\right\}$ is added to $\mathcal{D} i s p$, and the segment fails and is repeated.

4.3 The players invoke LC-Reconstruct to reconstruct $[r]$ (but skipping the first step, because shares of $[r]$ have already been broadcast).

4.4 Since the sharing $[r]$ was inconsistent, the invocation of LC-Reconstruct in the previous step will have located a new corrupt player, so the segment fails and is repeated.

5. Using the method for reconstructing secrets described in section 3.1, $P_{D}$ computes the random value $r$ associated with each of his $L$ input gates in this segment.

6. For each input gate with input $s$ and random sharing $[r], P_{D}$ broadcasts $s-r$.

7. For each $s-r$ broadcast in the previous step, each player locally computes $s-r+[r]$ (using the canonical sharing of $s-r$ as defined in section 3.1) as the sharing for that input gate. Since each player is storing each share as a sum of shares (one from each player), we update $[r]$ by adding the canonical sharing of $s-r$ to $\left[r^{(D)}\right]$ and leaving $\left[r^{(i)}\right]$ the same for $i \neq D$. In the dealer failed to broadcast a value for an input gate, or if the dealer was already in $\mathcal{C o r r}$, then the sharing for that gate is taken to be $[r]$.

\begin{tabular}{|l|cccc|}
\hline Input-Phase & $C B$ & $B C B$ & $C R$ & $B C R$ \\
\hline WithoutDispute & $n c_{I} d+n^{4} d+n^{5} \kappa \log d$ & $n^{2} c_{I}+n^{5}$ & $5 n^{2}$ & $4 n^{2}$ \\
PerDispute & $c_{I}+n^{2} \kappa \log d$ & $n^{2}+n d+d \log \mathcal{C}$ & 2 & $9+\log \mathcal{C}$ \\
\hline
\end{tabular}

\subsection{Computation Phase}

After the circuit preparation has been done and after the inputs have been provided by the players, the computation phase is just a matter of opening linear combinations of sharings and possibly resolving disputes.

Each affine gate is computed by performing local computations. Each multiplication gate is computed by opening affine combinations of known sharings. Each output gate is computed by publicly opening it. ${ }^{5}$ This means that the computation phase will consist of local operations and $c_{M}+c_{O}$ public openings.

The circuit will be divided into segments and evaluated one segment at a time. The segments will be constructed such that each segment has no more than $\left\lceil\left(c_{M}+c_{O}\right) / n^{2}\right\rceil$ gates, and a single segment only contains gates from one multiplicative layer of the circuit. This means that if $\mathcal{D}$ is the multiplicative depth of the circuit, then there are at most $n^{2}+\mathcal{D}$ segments. Each affine gate will be included in the first possible segment in which it can be evaluated.

If a fault occurs in some segment (which is to say that one of the opened sharings is inconsistent), then one or more new disputes are localized, and the segment is repeated.

It is important to remember that all sharings generated by VSS and Multiplication-Triple are sums of sharings such that one summand comes from each player. Since all sharings opened are affine combinations of these, this means that every sharing we will be opening in the computation phase is a sum of sharings with one summand coming from each player. Thus the protocol LC-Reconstruct can be performed.

\footnotetext{
${ }^{5}$ We assume that each player receives all the outputs, although the protocol could easily be modified to allow for private outputs.
} 


\section{Protocol: Computation-Phase}

For each segment with $L$ reconstructions, the following steps are executed. If one of the reconstructions is inconsistent, then a new dispute is found, and the segment is repeated.

1. For each affine gate in the segment, the players evaluate the gate by local computations.

2. The players invoke LC-Reconstruct multiple times in parallel for each output gate in the segment.

3. For each multiplication gate in the segment with inputs $[x]$ and $[y]$ and associated multiplication triple $([a],[b],[c])$, the following steps are performed in parallel.

3.1 In parallel with step 2, the players invoke LC-Reconstruct $([x-a])$ and LC-Reconstruct $([y-b])$.

3.2 The players assign the sharing $(x-a)(y-b)-(x-a)[b]-(y-b)[a]+[c]$ as the output of the gate.

\begin{tabular}{|l|cccc|}
\hline Computation-Phase & $C B$ & $B C B$ & $C R$ & $B C R$ \\
\hline WithoutDispute & 0 & $\mathcal{C} d$ & 0 & $\mathcal{D}$ \\
PerDispute & $\mathcal{C} n+n^{3}+n^{2} \kappa \log d$ & $n^{2}+n d+d \log \mathcal{C}$ & 2 & $6+\log \mathcal{C}$ \\
\hline
\end{tabular}

\subsection{Putting it All Together}

We perform the MPC protocol by invoking Preparation-Phase, Input-Phase, and Computation-Phase in succession. Note that there is a term $n$ added to the number of communication rounds to account for the fact that when a player is corrupted, all players will broadcast their shares sent by that player.

Theorem 1. A set of $n$ players communicating over a secure synchronous network can evaluate an agreed function of their inputs securely against an malicious, adaptive adversary with an arbitrary $Q^{2}$ adversary structure $\mathcal{A}$ with point-to-point communication bandwidth $O\left(n^{2} \mathcal{C} d+n^{4} d+n^{5} \kappa \log d\right)$ and broadcast bandwidth $O\left(n^{3} \mathcal{C}+n \mathcal{C} d+n^{5}+n^{3} d+n^{2} d \log \mathcal{C}\right)$, taking $20 n^{2}$ communication rounds and $27 n^{2}+\mathcal{D}+n^{2} \log \mathcal{C}$ broadcast rounds. Here, $d$ is the number of rows in the smallest multiplicative MSP representing $\mathcal{A}$, and $\kappa$ is the size of an element of $\mathbb{F}$.

\section{References}

[1] Donald Beaver and Avishai Wool. Quorum-based secure multi-party computation. In EUROCRYPT, pages 375-390, 1998.

[2] Zuzana Beerliová-Trubíniová and Martin Hirt. Efficient multi-party computation with dispute control. In $T C C$, pages 305-328, 2006.

[3] Ronald Cramer, Ivan Damågard, and Ueli Maurer. Span programs and general multi-party computation. Preliminary version appeared as BRICS tech. report, BRICS-RS-97-28, 1998.

[4] Ronald Cramer, Ivan Damgård, Stefan Dziembowski, Martin Hirt, and Tal Rabin. Efficient multiparty computations secure against an adaptive adversary. In EUROCRYPT, pages 311-326, 1999.

[5] Matthias Fitzi, Martin Hirt, and Ueli M. Maurer. General adversaries in unconditional multi-party computation. In ASIACRYPT, pages 232-246, 1999.

[6] Martin Hirt and Ueli Maurer. Complete characterization of adversaries tolerable in general multiparty computations. Proc. PODC, 1997. 
[7] Martin Hirt, Ueli M. Maurer, and Vassilis Zikas. Mpc vs. sfe : Unconditional and computational security. In ASIACRYPT, pages 1-18, 2008.

[8] Martin Hirt and Daniel Tschudi. Efficient general-adversary multi-party computation. In ASIACRYPT (2), pages 181-200, 2013.

[9] Mauricio Karchmer and Avi Wigderson. On span programs. In Structure in Complexity Theory Conference, pages 102-111, 1993.

[10] Ueli Maurer. Secure multi-party computation made simple. Discrete Applied Mathematics, 154(2):370 - 381, 2006. Coding and Cryptography.

[11] Adam Smith and Anton Stiglic. Multiparty computation unconditionally secure against $Q^{2}$ adversary structures. CoRR, cs.CR/9902010, 1999.

\section{A.1 Proofs}

Proof of Lemma 1. See [3] for the proof that the scheme is secure without the use of Kudzu shares (i.e., the case in which $r_{2}, \ldots, r_{e}$ are chosen completely randomly).

To see that using Kudzu shares does not compromise security, note that if two players are in dispute, then at least one of them is corrupt, so the adversary will know the zero-share anyway. Thus making the share public knowledge doesn't give the adversary any more information than he already had.

\section{Proof of Lemma 3.}

1. Assuming VSS terminates successfully:

1.1 We are guaranteed that there is at least one honest $P_{V} \notin \mathcal{D} i s p_{D}$ (because otherwise $P_{D}$ would be in $\mathcal{C}$ orr, and would not be sharing any values). So we are guaranteed that there is at least one random vector $\left(r_{1}, \ldots, r_{\ell}\right)$ such that $\sum_{k=1}^{\ell} r_{k}\left[s^{(k)}\right]+\left[u^{(V)}\right]$ will be opened toward an honest player. Let $R$ denote the subspace of $\mathbb{F}^{\ell}$ defined as the set of vectors $\left(r_{1}, \ldots, r_{\ell}\right)$ such that $\sum_{k=1}^{\ell} r_{k}\left[s^{(k)}\right]$ is a consistent sharing. ${ }^{6}$ Let $\mathbf{r}=\left(r_{1}, \ldots, r_{\ell}\right)$ be some vector such that $\sum_{k=1}^{\ell} r_{k}\left[s^{(k)}\right]+\left[u^{(V)}\right]$ is a consistent sharing. Then the set of all vectors $\left(r_{1}, \ldots, r_{\ell}\right)$ such that $\sum_{k=1}^{\ell} r_{k}\left[s^{(k)}\right]+\left[u^{(V)}\right]$ is a consistent sharing is $\mathbf{r}+R$.

Now if some $\left[s^{(k)}\right]$ is inconsistent, $R$ will be a proper subspace of $\mathbb{F}^{\ell}$. In this case, the probability that the sharing $\sum_{k=1}^{\ell} r_{k}\left[s^{(k)}\right]+\left[u^{(V)}\right]$ is inconsistent is $|\mathbf{r}+R| /\left|\mathbb{F}^{\ell}\right| \leq \kappa^{\ell-1} / \kappa^{\ell}=1 / \kappa$, which is negligible.

1.2 The correctness of the authentication tags follows from the correctness of Distribute-Tags.

2. The fact that any localized dispute is new follows from inspection of the protocol.

3. The only information the adversary receives are the corrupt players' shares of each $s^{(k)}$ and all the shares of $\sum_{k=1}^{\ell} r_{k}\left[s^{(k)}\right]+\left[u^{(V)}\right]$. Since the underlying secret sharing protocol is perfectly private (Lemma 1), the shares of $s^{(k)}$ give the adversary no information on $s^{(k)}$, and since the sharing $\left[u^{(V)}\right]$ is random, the shares of $\sum_{k=1}^{\ell} r_{k}\left[s^{(k)}\right]+\left[u^{(V)}\right]$ are independent of the $s^{(k)}$.

\section{Proof of Lemma 4.}

\section{Termination}

The fact that VSS satisfies the termination property of definition 1 follows from Lemma 3.

In VSS-Reconstruct, the players either execute step 6 or step 7. In the last sub-step of step 6 , if the shares of non-corrupt players are consistent, then the protocol terminates successfully, and otherwise the players move on to step 7 . So to prove that the honest players always complete VSS-Reconstruct, it suffices to

\footnotetext{
${ }^{6}$ It may be the case that $R=\{0\}$.
} 
prove the claim in the last sub-step of step 7 (which is sub-step 7.3); namely, we want to show that at step 7.3, any player who broadcast an incorrect share in the first step of VSS-Reconstruct will be in $\mathcal{C}$ orr . So suppose some $P_{i}$ sent an incorrect share in step 1. If $P_{i}$ sends the same share to some $P_{m} \notin \mathcal{D} i s p_{i}$ in step 7.1, then by Lemmas 2 and $3, P_{m}$ will label him corrupt. If $P_{i}$ sends a different share in step 7.1, then $P_{m}$ accuse $P_{i}$ in step 7.2. This means that any $P_{i}$ who broadcast an incorrect share in step 1 will now be in dispute will all honest players. Since the set of honest players is not in $\mathcal{A}$, this means that $P_{i}$ will be labeled corrupt. So only good shares are used in step 7.3, meaning that the protocol terminates successfully.

2. Privacy

Privacy of a secret $w$ before any execution of VSS-Reconstruct follows from Lemma 3. In addition, we need to show that invoking VSS-Reconstruct for some secret does not reveal any information about other secrets. This is because shares of other secrets are sent during invocations of Check-Message. However, note that whenever Check-Message is invoked, the person sending the shares is always in dispute with the dealer, (which will certainly be the case if the dealer is in $\mathcal{C}$ orr ). Since the dealer and the share-holder are in dispute, one of them is corrupt, so the adversary already knows all shares sent from the dealer to the share-holder, and revealing these shares to other players does not give the adversary any additional information.

3. Correctness

It follows from Lemma 3 that at the end of VSS, each of the sharings is consistent, which means that the vector of shares is in the span of the MSP matrix $M$. Any vector in the span of $M$ corresponds to a sharing of a unique value, and thus there is a unique value $r$ such that the vector of shares is a sharing of $r$. Furthermore, if the dealer is honest, then it is clear that $r$ is the value that was supposed to be shared.

Now we must verify that the honest players reconstruct $r$ at the end of VSS-Reconstruct. It is clear from inspection of VSS-Reconstruct that no matter how the protocol ends, the players always end up reconstructing from a consistent set of shares. Also note that this reconstruction uses the shares of all honest players (including Kudzu shares), because honest players broadcast correct shares in step 1, and even if the dealer accuses them of lying in step 3, they will be vindicated in step 6.4. Since the shares of honest players are sufficient to reconstruct a sharing, all honest players will reconstruct $r$.

Proof of Lemma 5. It is clear that if LC-Reconstruct succeeds, it will reconstruct the correct value, because in that case the shares broadcast in the first step were consistent. The fact that unsuccessful termination leads to finding a new corrupt player follows by examining steps 6.9 and 7.6.

To show that no information about any sharing other than $[w]$ is leaked to the adversary, note that in step 6, the only shares that are opened are shares held by a player that the dealer accuses of lying, and so the adversary already knew those shares; in step 7, the shares revealed are those dealt by a corrupt dealer, so again, the adversary learns nothing new.

Proof of Lemma 6 . Since at least one player is honest, at least one summand vector $s^{(1, i)}, \ldots, s^{(\ell, i)}$ will be completely random. Thus the sum vector will be random.

The only way in which Generate-Randomness might terminate unsuccessfully is if VSS or LC-Reconstruct terminates unsuccessfully, in which case a new dispute will be found.

Proof of Lemma \%. That a new dispute is localized when Multiplication-Triple terminates unsuccessfully is clear from inspection of the protocol.

Privacy of $a^{(k)}$ follows from the fact that it is a sum of sharings, one sharing coming from each player. The only time that a sharing involving $a^{(k)}$ is opened is in steps 1.3 and 2.4, and in both cases it is masked (with $\left[r^{(i, k)}\right]\left[1^{(i, k)}\right]$ and $\left[\widetilde{c}^{(i, k)}\right]$, respectively). 
Privacy of $b^{(k)}$ follows from the fact that it is a sum of sharings, one sharing coming from each player. Sharings involving each of the summands $b^{(i, k)}$ are only opened in steps 1.3 and 2.2 ; in step 1.3, the sharing is opened to the player who generated it, and in step 2.2, the sharing is masked by $\widetilde{b}^{(i, k)}$.

Privacy of $c^{(k)}$ follows from the fact that it is a sum of sharings, one sharing coming from each player, and that the only time that a sharing involving a $c^{(i, k)}$ is opened is in step 2.4, where it is masked with $\widetilde{c}^{(i, k)}$.

With overwhelming probability, the invocations of VSS used to construct the sharings in the first step generate valid sharings, and since $a^{(k)}, b^{(k)}$, and $c^{(k)}$ are affine combinations of these sharings, they are valid sharings. In order to show that $c^{(k)}=a^{(k)} b^{(k)}$, it suffices to show that $c^{(i, k)}=a^{(k)} b^{(i, k)}$ for each $i$. In particular, we want to show that if $z^{(i, k)}$ reconstructed in step 2.4 is zero, then with overwhelming probability, $c^{(i, k)}=a^{(k)} b^{(i, k)}$. Note that $z^{(i, k)}=0$ if and only if $s^{(k)}\left(c^{(i, k)}-a^{(k)} b^{(i, k)}\right)=-\left(\widetilde{c}^{(i, k)}-\widetilde{a}^{(k)} \widetilde{b}^{(i, k)}\right)$. If $c^{(i, k)}-a^{(k)} b^{(i, k)} \neq 0$, then since $s^{(k)}$ is random, $z^{(i, k)}$ could only be zero with negligible probability.

\section{A.2 The Information Checking Protocols}

We now give the information checking protocols explicitly.

Protocol: Distribute-Tags $\left(P_{S}, P_{R}, P_{V},\left(m^{(1)}, \ldots, m^{(\ell)}\right)\right)$

We call the sender $P_{S}$, the receiver $P_{R}$, and the verifier $P_{V}$. We assume that a vector of messages $\mathbf{m}=$ $\left(m^{(1)}, \ldots, m^{(\ell)}\right) \in \mathbb{F}^{\ell}$ (with $\ell \leq d$ ) has already be sent from the $P_{S}$ to $P_{R}$. We also assume there is an extension field $\mathbb{G}$ of $\mathbb{F}$ such that $\mathbb{G}$ has minimal size subject to $|\mathbb{G}| \geq d|\mathbb{F}|$. (The field $\mathbb{G}$ is fixed throughout the entire MPC protocol.)

1. Generating Tags:

$1.1 P_{S}$ picks $2 \kappa$ random elements $y_{1}, \ldots, y_{\kappa}, u_{1}, \ldots, u_{\kappa} \in \mathbb{G}$.

1.2 For each $i=1, \ldots, \kappa, P_{S}$ determines $v_{i}$ such that the points $\left(0, y_{i}\right),\left(1, m^{(1)}\right), \ldots,\left(\ell, m^{(\ell)}\right),\left(u_{i}, v_{i}\right)$ lie on a degree $\ell$ polynomial (over $\mathbb{G}$ ).

$1.3 P_{S}$ sends the authentication tags $y_{1}, \ldots, y_{\kappa}$ to $P_{R}$ and the verification tags $z_{i}=\left(u_{i}, v_{i}\right)$ to $P_{V}$ for each $i$.

2. Fault Detection:

2.1 $P_{V}$ partitions the set $\{1, \ldots, \kappa\}$ into sets $I$ and $\bar{I}$ of almost equal size $(|| I|-| \bar{I}|| \leq 1)$ and sends $\left\{z_{i}\right\}_{i \in I}$ to $P_{R}$.

2.2 $P_{R}$ checks for each $z_{i}$ sent by $P_{V}$ that the points $\left(0, y_{i}\right),\left(1, m^{(1)}\right), \ldots,\left(\ell, m^{(\ell)}\right),\left(u_{i}, v_{i}\right)$ lie on a polynomial of degree $\ell$. If any one of these checks fails, $P_{R}$ broadcasts (reject). Otherwise, $P_{R}$ broadcasts (accept).

3. Fault Localization:

If $P_{R}$ broadcasted (reject) above, then the following steps are executed.

$3.1 P_{R}$ picks one $z_{i}$ that failed the check in step 2.2 and broadcasts $\left(i, s, z_{i}\right)$.

3.2 $P_{V}$ and $P_{S}$ broadcast $z_{i}$.

3.3 If the value broadcasted by $P_{S}$ and $P_{V}$ differ, then $\left\{P_{S}, P_{V}\right\}$ is added to $\mathcal{D} i s p$. If the value broadcasted by $P_{R}$ and $P_{V}$ differ, then $\left\{P_{R}, P_{V}\right\}$ is added to $\mathcal{D} i s p$. Otherwise, $\left\{P_{R}, P_{S}\right\}$ is added to Disp.

\begin{tabular}{|l|cccc|}
\hline Distribute-Tags & $C B$ & $B C B$ & $C R$ & $B C R$ \\
\hline WithoutDispute & $\kappa \log d$ & 1 & 2 & 1 \\
PerDispute & 0 & $\log d$ & 0 & 2 \\
\hline
\end{tabular}


In the execution of the protocol, a dispute may arise between the sender and receiver as to what the value of the vector of messages was. The vector is revealed to the verifier using Check-Message, and then the verifier either confirms or denies what the receiver claims to have received.

Protocol: Check-Message $\left(P_{R}, P_{V},\left(m^{(1)}, \ldots, m^{(\ell)}\right)\right)$

1. The receiver sends the vector of messages $\mathbf{m}=\left(m^{(1)}, \ldots, m^{(\ell)}\right)$ along with authentication tags $\left\{y_{i}\right\}_{i \in \bar{I}}$ to $P_{V}$.

2. The verifier checks that the points $\left(0, y_{i}\right),\left(1, m^{(1)}\right), \ldots,\left(\ell, m^{(\ell)}\right),\left(u_{i}, v_{i}\right)$ all lie on a polynomial of degree $\ell$ for each $i=\bar{I}$. If any one of these $|\bar{I}|$ checks passes, then the verifier broadcasts (accept). Otherwise, the verifier broadcasts (reject).

\begin{tabular}{|l|cccc|}
\hline Check-Message & $C B$ & $B C B$ & $C R$ & $B C R$ \\
\hline WithoutDispute & $\ell+\kappa \log d$ & 1 & 1 & 1 \\
PerDispute & 0 & 0 & 0 & 0 \\
\hline
\end{tabular}

Proof of Lemma 2. The proof is the same as in [2] with the exception of claim 3, whose proof is as follows: The probability that $P_{R}$ could guess a point $\left(0, y_{i}\right)$ that worked for some $\mathbf{m}^{\prime} \neq \mathbf{m}$ is no more than $\kappa /(|\mathbb{G}|-$ $\ell-1) \leq \kappa /\left(d 2^{\kappa}-\ell-1\right) \leq \kappa /\left(d\left(2^{\kappa}-1\right)-1\right)$, which is negligible.

\section{A.3 The VSS Reconstruct Protocol}

\section{Protocol: VSS-Reconstruct $([w])$}

We assume that the sharing $[w]$ has been shared by a dealer $P_{D}$ using VSS.

1. Each player not in $\mathcal{C}$ orr that holds a non-Kudzu share of $[w]$ broadcasts their share.

2. If the shares broadcast in the previous step and the Kudzu shares form a consistent sharing (that is, they are in the span of $M_{\mathcal{P}-\mathcal{C} \text { orr }}$ ), then the secret $w$ is reconstructed as described in section 3.1, and the protocol terminates.

3. If the shares broadcast in step 1 form an inconsistent sharing, then $P_{D}$ broadcasts the index $i$ of each player he accuses of sending an incorrect share.

4. If $P_{D}$ did not broadcast an index in the previous step, then $P_{D}$ is added to $\mathcal{C}$ orr (if he is not already in Corr $)$. Also, if we remove the shares $P_{D}$ accused of being corrupted, and the remaining shares are still inconsistent, then $P_{D}$ is added to $\mathcal{C}$ orr. Lastly, if the set of players in dispute with $P_{D}$ is no longer in $\mathcal{A}$, then $P_{D}$ is added to $\mathcal{C}$ orr.

5. If $P_{D} \notin \mathcal{C}$ orr, then proceed to step 6 . Otherwise, proceed to step 7 .

6. Dealer not in $\mathcal{C}$ orr

6.1 For each player $P_{i}$ accused by $P_{D}$ in step 3, the players invoke Check-Message $\left(P_{i}, P_{m}, \mathbf{s}_{i}\right)$ for each player $P_{m} \notin \mathcal{D} i s p_{i} \cup \mathcal{D} i s p_{j}$, where $\mathbf{s}_{i}$ is the vector defined in step 1.2 of the invocation of VSS in which $[w]$ was shared.

6.2 For any $P_{i}$ who sent a share to $P_{m}$ that was different than the share broadcast in step $1, P_{m}$ broadcasts (accuse, $i$ ), and $\left\{P_{m}, P_{i}\right\}$ is added to $\mathcal{D} i s p$. 
6.3 For each $P_{m} \notin \mathcal{D} i s p_{i}$ that rejected the message sent by $P_{i}$ in the invocation of Check-Message, $\left\{P_{i}, P_{m}\right\}$ is added to $\mathcal{D} i s p$. For each $P_{m}$ that accepted the message, $\left\{P_{D}, P_{m}\right\}$ is added to Disp.

6.4 At this point, any $P_{i}$ who was accused by $P_{D}$ and who broadcast an incorrect share in step 1 will have been accused by all honest $P_{m} \notin \mathcal{D} i s p_{i}$, meaning that $P_{i}$ will be added to $\mathcal{C}$ orr. Similarly, if $P_{i}$ was accused by $P_{D}$ but broadcast a correct share in step 1 , then $P_{D}$ will have been accused by all honest $P_{m} \notin \mathcal{D} i s p_{D}$, meaning that $P_{D}$ will be added to $\mathcal{C}$ orr .

6.5 If the shares of players not in $\mathcal{C}$ orr (together with the Kudzu shares) form a consistent sharing, then those shares are used to reconstruct $w$. If those shares are inconsistent, then the dealer is added to Corr, and the players proceed to step 7.

7. Dealer in $\mathcal{C}$ orr

7.1 For all $P_{i}$ that do not hold Kudzu-shares and for all $P_{m} \notin \mathcal{D} i s p_{i}$, the players invoke Check-Message $\left(P_{i}, P_{m}, \mathbf{s}_{i}\right)$, where $\mathbf{s}_{i}$ is the vector defined in step 1.2 of the invocation of VSS in which $[w]$ was shared.

7.2 For any $P_{i}$ who sent a share to $P_{m}$ that was different than the share broadcast in step $1, P_{m}$ broadcasts (accuse, $i$ ), and $\left\{P_{m}, P_{i}\right\}$ is added to $\mathcal{D} i s p$.

7.3 At this point, any $P_{i}$ who broadcast an incorrect share in step 1 will have been accused by all $P_{m} \notin \mathcal{D} i s p_{i}$, meaning that $P_{i}$ will be added to $\mathcal{C}$ orr . The shares of players not in $\mathcal{C}$ orr are now used to reconstruct $w$.

\begin{tabular}{|l|cccc|}
\hline VSS-Reconstruct & $C B$ & $B C B$ & $C R$ & $B C R$ \\
\hline WithoutDispute & 0 & $d$ & 0 & 1 \\
PerDispute & $n^{2} \ell+n^{2} \kappa \log d$ & $n^{2}$ & 2 & 5 \\
\hline
\end{tabular}

